Rotaviren, Varizellen und Pertussis

\section{Welchen Stellenwert haben Impfungen?}

- Infektionen mit Rotaviren (RV) sind die häufigste Ursache schwerer Durchfallerkrankungen bei Kindern unter fünf Jahren. Bis zum dritten Geburtstag hat sich nahezu jedes Kind schon einmal mit diesen hoch kontagiösen Erregern infiziert. „Die Kleinsten trifft es meist am härtesten", betonte Prof. Jim Gray, East-Anglia/Norwe-

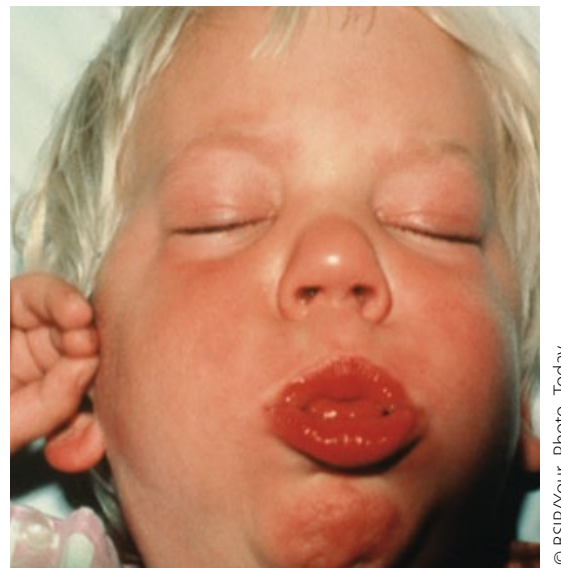

Keuchhusten ist nicht nur eine Kinderkrankheit. gen. Erbrechen und häufige wässrige Durchfälle führen bei Säuglingen und Kleinkindern rasch zur lebensbedrohlichen Dehydrierung und erfordern deshalb oft eine stationäre Aufnahme. Seit Einführung der Schluckimpfung sei die Hospitalisierungsrate weltweit um bis zu $93 \%$ gesunken. Der humane attenuierte Lebendimpfstoff (Rotarix ${ }^{\mathrm{TM}}$ ) imitiert die natürliche Infektion und schützt vor allem vor schweren Krankheitsverläufen. Die Impfserie besteht aus zwei Dosen (Beginn ab der sechsten Woche) und sollte vorzugsweise bis zur 16 . Lebenswoche abgeschlossen sein

\section{Pertussis: Kokonstrategie schützt Neugeborene}

Keuchhusten ist nicht nur eine Kinderkrankheit, führte Prof. John Roord, Amsterdam/Niederlande, aus. In den vergangenen Jahren sei eine zunehmende Verschiebung in das Jugend- und Erwachsenenalter beobachtet worden. „In dieser Altersgruppe verläuft die Infektion oft atypisch und wird häufig nicht erkannt." Er- krankte Familienmitglieder stellen deshalb eine Gefahr für Neugeborene dar, die selbst noch nicht geimpft werden können. Die STIKO empfiehlt deshalb Frauen im gebärfähigen Alter mit Kinderwunsch und Betreuungspersonen wie Familienmitglieder im Umfeld eines Neugeborenen eine Auffrischung (z. B. mit dem Tdap-Dreifachimpfstoff Boostrix ${ }^{T M}$ ) im Sinne einer Kokonstrategie.

\section{Varizellen: Votum für Zweitimpfung}

Dass auch harmlos anmutende Kinderkrankheiten wie Windpocken mitunter zu ernsthaften Komplikationen führen können, berichtete Prof. Vana Papaenvangelou, Athen/Griechenland. Zur Reduktion von Varizellendurchbrüchen habe sich eine zweite Impfdosis bewährt (z. B. mit dem Vierfachimpfstoff Priorix-Tetra ${ }^{\mathrm{TM}}$ gegen Masern, Mumps, Röteln und Varizellen).

- Dr. Martina-Jasmin Utzt

Quelle: Satellitensymposium „Successes and challenges in paediatrics with varicella, rotavirus and pertussis vaccination", 30 . Kongress der European Society of Paediatric Infectious Diseases (ESPID), Thessaloniki/Griechenland, Mai 2012 (Veranstalter: GSK)

\title{
Typ-2-Diabetes
}

\section{Mit GLP-1-Analogon mehrere Therapieziele im Blick}

— Eine optimale Therapie bei Typ-2-Diabetes sollte Prof. Werner Kern, Ulm, zufolge, Mehreres bieten: Gute glykämische Kontrolle über einen langen Zeitraum bei geringem Hypoglykämie-Risiko, Verbesserung der Betazellfunktion, klinisch relevante Gewichtsabnahme, Optimierung weiterer signifikanter kardiovaskulärer Risikofaktoren, die den Diabetes begleiten (z.B. Bluthochdruck und Dyslipidämie), einfache und flexible Behandlungsregime.

Wie sich in den letzten Jahren zeigte, können GLP-1-basierte Behandlungsregime einem suboptimalen Management des Typ-2-Diabetes gegensteuern. Für das humane GLP-1-Analogon Victoza ${ }^{\circledR}$ (Liraglutid) wurde in einer retrospektiven Analyse von Studiendaten an 1530 Diabetikern belegt: Nach 26 Wochen reduzierte sich der
$\mathrm{HbA}_{1 c}$-Wert unter 1,8 mg Liraglutid im Vergleich zum Ausgangswert im Mittel um $1,5 \%$, um $1,24 \%$ unter $1,2 \mathrm{mg}$ Liraglutid und um 0,9\% unter Sitagliptin. Darüber hinaus erreichten unter Liraglutid signifikant mehr Patienten den $\mathrm{HbA}_{1 \mathrm{c}}$-Zielwert von $\leq 7 \%$ (Pratley R et al. Lancet 2010; 375 (9724): 1447-1456). Nach 52 Wochen erzielten, verglichen mit Sitagliptin, etwa doppelt so viele Patienten unter Liraglutid $(1,2 \mathrm{mg})$ den kombinierten Endpunkt: $\mathrm{HbA}_{1 \mathrm{c}}$-Wert $<7 \%$, keine Gewichtszunahme und keine Hypoglykämien (Pratley R et al. Int J Clin Pract 2011; 65: 397-407).

Besondere Bedeutung für die Effektivität der antidiabetischen Therapie scheint der Zeitpunkt des Behandlungsbeginns zu haben. Die besten Ergebnisse mit Liraglutid wurden erzielt, wenn der $\mathrm{HbA}_{1 c}$-Basis- wert unter 8,5\%, die Dauer des Typ-2-Diabetes unter 4,9 Jahre lag und die bisherige Therapie aus einem oralen Antidiabetikum oder Ernährungsumstellungen bestand. Unter diesen Voraussetzungen erreichten die meisten Patienten mit dem humanen GLP-1-Analogon den kombinierten Endpunkt $\mathrm{HbA}_{1 \mathrm{c}}$ unter $7 \%$, ohne Gewichtszunahmen und ohne Hypoglykämien, wie die gepoolte Analyse der 26-Wochen-Daten von sieben Phase-3-Studien mit Liraglutid $(1,8 \mathrm{mg}$ ) zeigte (Ratner $\mathrm{R}$ et al. Diabetologia 2012; 55 (Suppl1): S332).

- Dr. Jochen Aumiller

Quelle: Prof. Werner Kern, UIm, in „Meet the Expert: Worauf es beim erfolgreichen Einsatz des humanen GLP-1-Analogons ankommt", EASD-Kongress, Berlin, Oktober 2012 (Veranstalter: Novo Nordisk) 